\title{
Improved Detection of Foreign Bodies on Radiographs Using X-ray Dark-Field and Phase-Contrast Imaging
}

\author{
Katharina Hellbach, $M D,{ }^{*}$ Ebba Beller, MD, $†$ Andreas Schindler, MD, * Franziska Schoeppe, MD, * \\ Nina Hesse, MD, * Alena Baumann, MD, * Regina Schinner, Dipl-Stat, * Sigrid Auweter, PhD, * \\ Christian Hauke, MSc, $\$$ Marcus Radicke, PhD, $\$$ and Felix G. Meinel, MD $†$
}

\begin{abstract}
Purpose: The aim of this study was to investigate whether the detection of foreign bodies can be improved using dark-field and phase-contrast radiography compared with conventional (transmission) radiographs.

Materials and Methods: Experiments were performed using ex vivo pig paws, which were prepared with differently sized foreign bodies of metal, wood, and glass $(\mathrm{n}=10$ each). Paws without foreign bodies served as controls $(\mathrm{n}=30)$. All images were acquired using an experimental grating-based large object radiography system. Five blinded readers (second- to fourth-year radiology residents) were asked to assess the presence or absence of any foreign body. Sensitivity and specificity for the detection of metal, wood, glass, and any foreign body were calculated and compared using McNemar test and generalized linear mixed models. Results: Sensitivity for the detection of metal foreign bodies was $100 \%$ for all readers and image combinations. The sensitivity for the detection of wooden foreign bodies increased from $2 \%$ for transmission images to $78 \%$ when dark-field images were added $(P<0.0001)$. For glass foreign bodies, sensitivity increased from $84 \%$ for transmission images to $96 \%$ when adding phase-contrast images $(P=0.041)$. Sensitivity for the detection of any foreign body was $91 \%$ when transmission, dark-field, and phase-contrast images were viewed simultaneously, compared with $62 \%$ for transmission images alone $(P<0.0001)$. Specificity was $99 \%$ to $100 \%$ across all readers and radiography modalities.

Conclusions: Adding dark-field images substantially improves the detection of wooden foreign bodies compared with the analysis of conventional (transmission) radiographs alone. Detection of glass foreign bodies was moderately improved when adding phase-contrast images.
\end{abstract}

Key Words: foreign body detection, radiography, dark-field imaging, phase-contrast imaging

(Invest Radiol 2018;53: 352-356)

atients with skin and soft tissue wounds are commonly seen in the emergency department. ${ }^{1}$ In $7 \%$ to $15 \%$ of these cases, retained foreign bodies can be found in the wounds. ${ }^{2,3}$ Despite thorough examination, up to $38 \%$ of foreign bodies are overseen. ${ }^{4}$ These missed foreign bodies can lead to complications such as persistent pain, infections, impaired wound healing, and loss of function. ${ }^{5}$ Most commonly, retained foreign bodies are made of metal, glass, or wood. ${ }^{6}$ Although patients typically undergo physical examination and radiography, glass is missed inside the wounds in approximately $50 \%$ of the cases. ${ }^{7}$ Less than $10 \%$ of wooden foreign bodies can be identified using plain radiographs. ${ }^{6}$ Unlike metal objects, glass and wood can be radiolucent, making detection of such foreign bodies on radiographs rather difficult or even impossible. ${ }^{8}$

Received for publication November 23, 2017; and accepted for publication, after revision, December 28, 2017

From the *Department of Radiology, University Hospital, LMU Munich, Munich; †Department of Radiology, Rostock University Medical Center, Rostock; and Siemens Healthcare GmbH, Forchheim, Germany.

Conflicts of interest and sources of funding: Marcus Radicke and Christian Hauke are employees at Siemens Healthcare. All other authors declare no conflicts of interest. Correspondence to: Katharina Hellbach, MD, Department of Radiology, University Hospital, LMU Munich, Marchioninistr.15, 81377 Munich, Germany. E-mail: katharina.hellbach@med.uni-muenchen.de.

Copyright (C) 2018 Wolters Kluwer Health, Inc. All rights reserved.

ISSN: 0020-9996/18/5306-0352

DOI: 10.1097/RLI.0000000000000450
Ultrasonography is occasionally used for the detection of foreign bodies but is only moderately sensitive and strongly dependent on the examiner's skills. ${ }^{4}$

Dark-field and phase-contrast imaging might offer a potential solution to this problem. By introducing a 3-grating Talbot-Lau interferometer into the $\mathrm{x}$-ray beam, this novel $\mathrm{x}$-ray imaging modality allows to reconstruct 3 different images from a single radiograph. In addition to a standard $\mathrm{x}$-ray image (which visualizes relative transmission of the x-ray beam through tissues), dark-field and differential phase-contrast images are obtained by applying Fourier analysis to the raw data. ${ }^{9-11}$ This technique has previously been explored in pulmonary ${ }^{12,13}$ and cardiovascular ${ }^{14,15}$ imaging. The dark-field image visualizes small-angle scattering of x-rays within tissue. ${ }^{16}$ Because of its fibrous structure, wood generates a strong dark-field signal. ${ }^{17}$ This makes dark-field imaging a promising technique for the detection of wooden foreign bodies. Phase-contrast images visualize the phase-shift of $\mathrm{x}$-ray beams within tissue with enhanced representation of edges, ${ }^{18}$ which might facilitate identifying glass fragments.

The purpose of this study was to investigate whether adding dark-field and phase-contrast imaging to conventional transmission radiographs will lead to an improved detection of foreign bodies.

\section{MATERIALS AND METHODS}

\section{Sample Preparation}

Experiments were performed using ex vivo pig paws purchased at a local butcher's shop. The paws were prepared with differently sized foreign bodies of metal, wood, and glass $(\mathrm{n}=10$ each, sizes ranging between $3 \mathrm{~mm}$ and $3 \mathrm{~cm}$, Fig. 1) by placing the foreign bodies on the dorsal surface of the paws. Paws without foreign bodies served as controls $(n=30)$.

\section{Prototype X-ray Dark-Field and Phase-Contrast Scanner}

Experiments were carried out with a Siemens Gigalix x-ray tube (Siemens Healthcare GmbH, Erlangen, Germany). The tube has a tungsten anode $(\mathrm{W})$ with a filtration of $0.3 \mathrm{~mm}$ of copper $(\mathrm{Cu})$. The focal spot size is $0.4 \mathrm{~mm}$ (IEC 60336). Gratings were fabricated by the LIGA process of the Karlsruhe Institute of Technology (KIT). The grating size of $70 \mathrm{~mm}$ diameter is limited by the wafer size. We restricted the active area per projection to $20 \times 20 \mathrm{~mm}^{2}$ to avoid artifacts due to planar gratings in a cone beam geometry. The grating periods are $\mathrm{p}_{0}=11.54 \mu \mathrm{m}$, $\mathrm{p}_{1}=3.39 \mu \mathrm{m}$, and $\mathrm{p}_{2}=4.8 \mu \mathrm{m}$. The heights of the gratings $\mathrm{G} 0$ and $\mathrm{G} 2$ are $275 \mu \mathrm{m}$ and $190 \mu \mathrm{m}$, respectively. The G1 height was $6.37 \mu \mathrm{m}$, imposing a phase shift of $\pi / 2$ at $62.5 \mathrm{keV}$ to the $\mathrm{x}$-ray wave front. The corresponding distances for the Talbot-Lau interferometer were G0-G1 $=981 \mathrm{~mm}$ and G1-G2 $=410 \mathrm{~mm}$. Images were recorded with a Xineos $1515 \mathrm{C}$ flat panel detector (Teledyne DALSA, Waterloo, Canada). We used a region of interest of $800 \times 800$ pixels with a pixel pitch of $99 \mu \mathrm{m}$ (Fig. 2). 


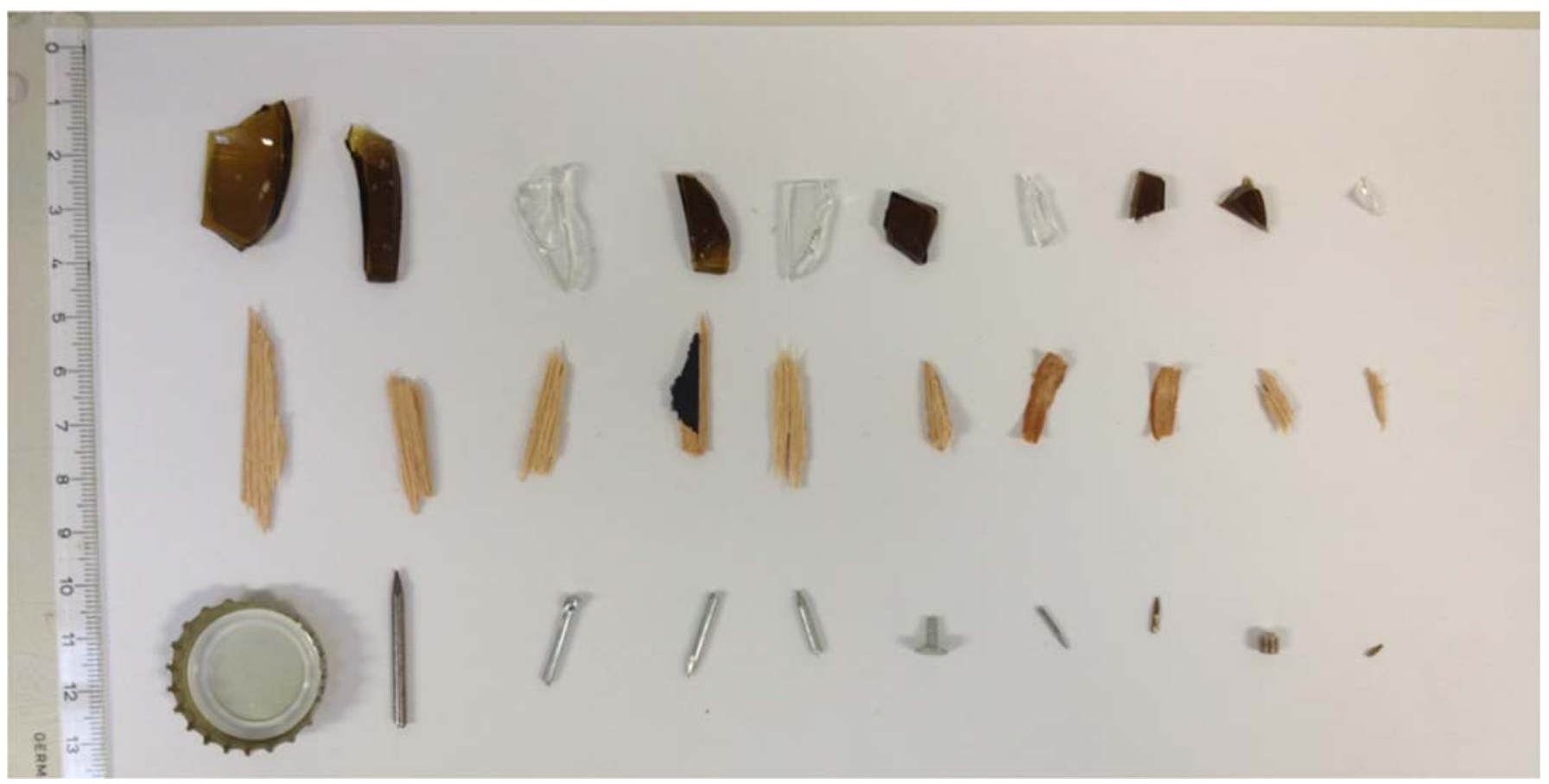

FIGURE 1. The foreign bodies used in this study are shown ordered by size; glass (upper row), wood (middle row), and metal (lower row).

\section{Imaging Protocol}

The field of view (FOV) of 1 image tile is $2 \times 3 \mathrm{~cm}^{2}$, determined by the size of the gratings. The sampling of the full object was done by line wise scanning of the object. By stitching the acquired tiles, a maximum FOV of $120 \times 30 \mathrm{~cm}^{2}$ can be generated. To depict a pig paw, a total of 6 tiles had to be scanned, resulting in an imaging time of approximately 3 minutes. The scanner was operated with 8 phase-stepping

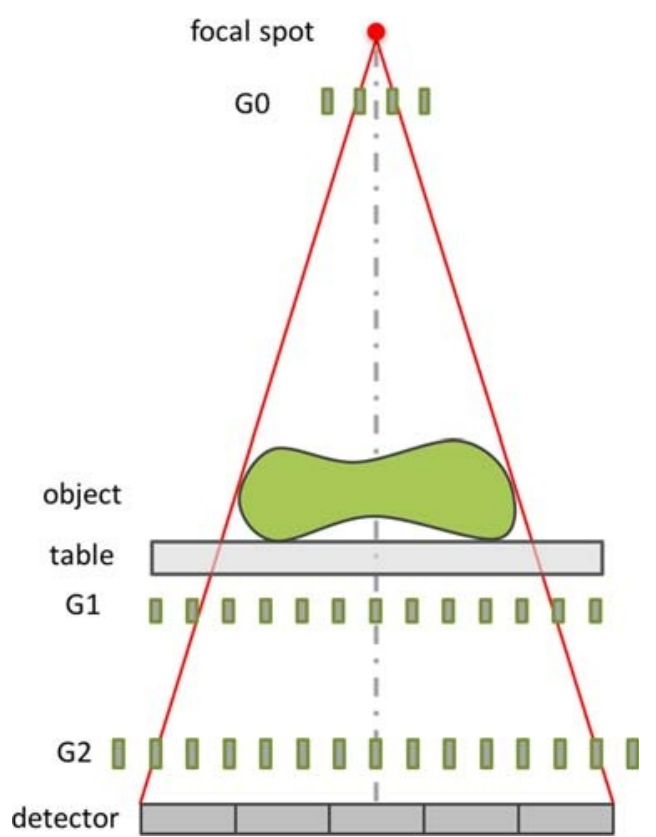

FIGURE 2. Schematic drawing of the phase-contrast scanning system used in this study. positions of the analyzer grating. Scanning parameters were set to $60 \mathrm{kV}, 320 \mathrm{~mA}$, and 50 milliseconds pulse time. Reconstruction and postprocessing of the acquired data were performed using a combination of $\mathrm{C}++$ and Matlab (Mathworks Inc, MA) software packages. The air kerma (skin entrance dose to the object) was measured with a PTW NOMEX (PTW, Freiburg, Germany) dosimeter. Images were acquired with an applied skin entrance air kerma of $0.19 \mathrm{mGy}$.

\section{Reader Study}

Five readers (second- to fourth-year radiology residents, initials blinded) were asked to independently assess the presence or absence of any foreign body. A total of 4 rounds of readings were performed in the following order:

1. transmission images alone

2. transmission and dark-field images

3. transmission and phase-contrast images

4. transmission, dark-field, and phase-contrast images.

A 2-week interval was required between rounds 2 and 3 to minimize recall bias. Before the readings started, all readers attended a briefing session explaining the basic principles of dark-field and phase-contrast imaging, including several image examples (not taken from this study). All images were presented as DICOM files in a commercially available DICOM reader (OsiriX MD; version 3.0.2, Pixmeo, Bernex, Switzerland), and readers were encouraged to freely adjust the window settings for optimal assessment.

\section{Statistical Analysis}

The statistical analysis was performed by an independent statistician (initials blinded) using SAS Version 9.4 for Windows (Copyright SAS Institute Inc, Cary, NC). Interreader agreement for the detection of foreign bodies was determined using Fleiss kappa statistics comparing multiple raters for nominal response. ${ }^{19}$ General linear mixed models 
were calculated with the combination of images as a fixed effect and the individual readers as a random effect to test for differences in sensitivity and specificity.

\section{RESULTS}

\section{Interreader Agreement}

Across all readers and samples, there was excellent interreader agreement for the detection of foreign bodies (Fleiss kappa $0.94 \pm 0.02$, $P<0.0001)$.

\section{Sensitivity for Metal Foreign Bodies}

Metal foreign bodies were visible in all image modalities (Fig. 3). Sensitivity for the detection of metal foreign bodies was $100 \%$ for all readers and all image combinations (Table 1 ).

\section{Sensitivity for Wooden Foreign Bodies}

Wooden foreign bodies were best visualized in dark-field images (Fig. 3). The sensitivity for the detection of wooden foreign bodies was as low as $2 \%$ for transmission images alone and significantly increased to $78 \%$ when dark-field images were added $(P<0.0001$, Table 1$)$. Adding phase-contrast images to transmission images moderately improved sensitivity for wooden foreign bodies (from $2 \%$ to $16 \% ; P=0.0264$ ). The combination of all 3 images was as good as the combination of transmission and dark-field images ( $78 \%$ sensitivity for both, $P=1.0000)$.

\section{Sensitivity for Glass Foreign Bodies}

Glass foreign bodies were best visualized in phase-contrast images (Fig. 3). The sensitivity for the detection of glass foreign bodies was $84 \%$ for transmission images alone and increased to $96 \%$ when adding phase-contrast images $(P=0.041$, Table 1$)$. Adding dark-field images to transmission images did not significantly improve sensitivity
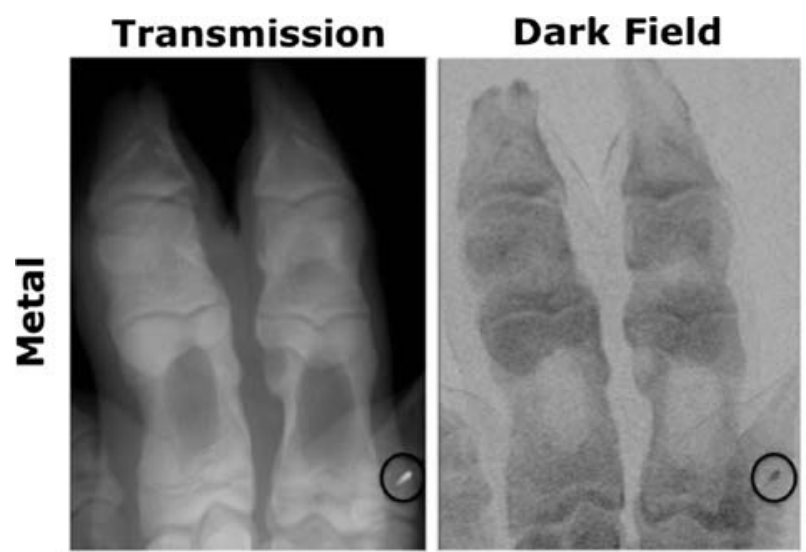

\section{Phase Contrast}
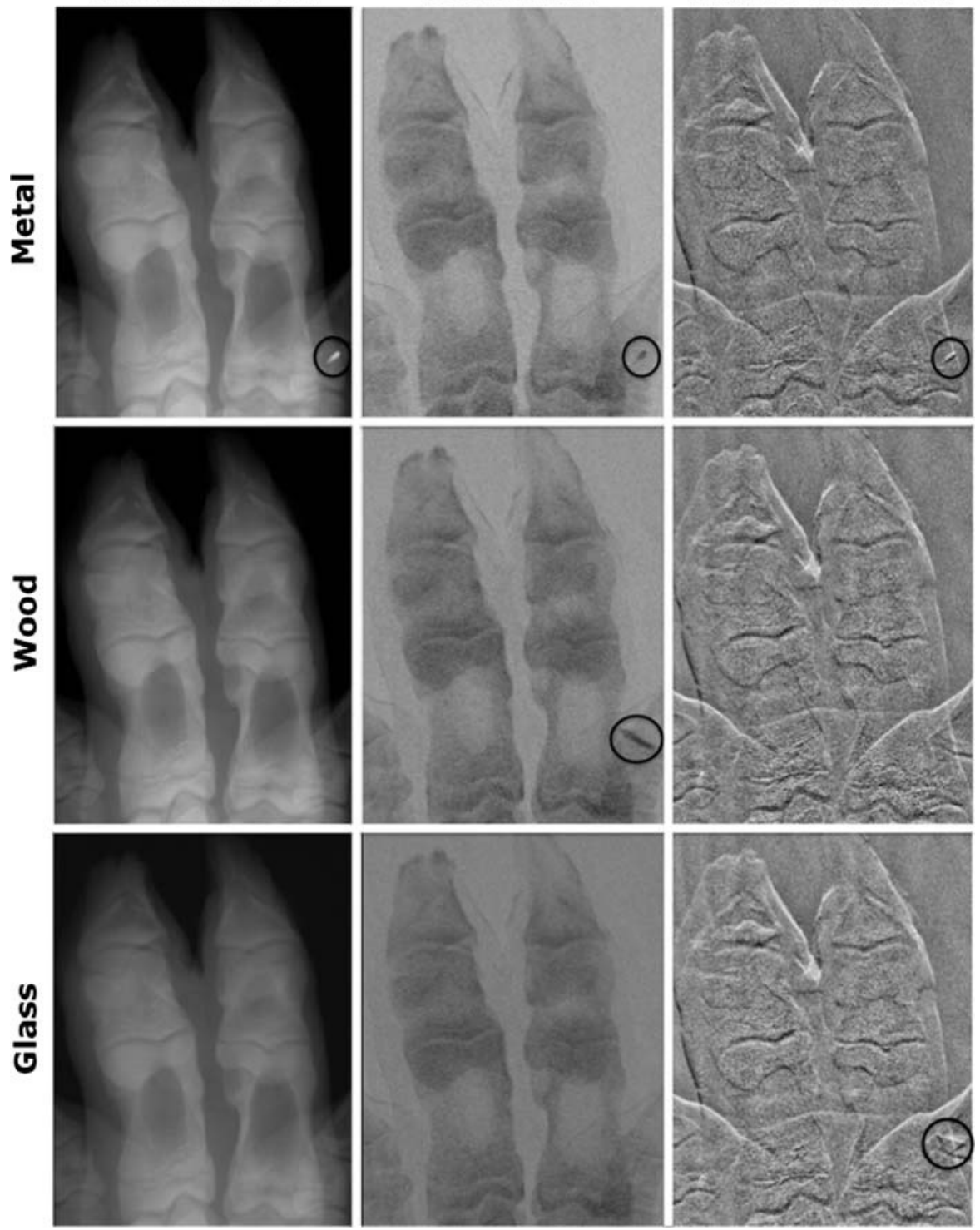

FIGURE 3. Examples of transmission (left), dark-field (middle), and phase-contrast (right) images equipped with foreign bodies made of metal (upper row), wood (middle row), and glass (lower row). The images in each row are different reconstructions from the same acquisition. As indicated by the black circles, wood is best seen on dark-field images, glass is best seen on phase-contrast images, and metal is clearly visible on all 3 images. 
TABLE 1. Sensitivity and Specificity

\begin{tabular}{|c|c|c|c|c|c|c|c|c|c|c|}
\hline \multirow[b]{3}{*}{$\mathrm{T}$} & \multicolumn{8}{|c|}{ Sensitivity in \% $[95 \% \mathrm{CI}]$} & & \\
\hline & \multicolumn{2}{|c|}{ Metal } & \multicolumn{2}{|c|}{ Wood } & \multicolumn{2}{|c|}{ Glass } & \multicolumn{2}{|c|}{ All Foreign Bodies } & \multicolumn{2}{|c|}{ Specificity in \% $[95 \% \mathrm{CI}]$} \\
\hline & 100 & [93-100] & 2 & {$[0-11]$} & 84 & [71-93] & 62 & [54-70] & 99 & [96-100] \\
\hline $\mathrm{T}+\mathrm{D}$ & 100 & [93-100] & 78 & [64-88] & 88 & [76-95] & 89 & [82-93] & 100 & [98-100] \\
\hline $\mathrm{T}+\mathrm{P}$ & 100 & [93-100] & 16 & [7-29] & 96 & [86-100] & 71 & [63-78] & 99 & [95-100] \\
\hline $\mathrm{T}+\mathrm{D}+\mathrm{P}$ & 100 & [93-100] & 78 & [64-88] & 94 & [83-99] & 91 & {$[85-95]$} & 99 & [95-100] \\
\hline
\end{tabular}

Sensitivity and specificity for the detection of foreign bodies of transmission images (T) alone as well as in combination with dark-field (D), phase-contrast (P) images, or both are shown.

$\mathrm{CI}$ indicates confidence interval.

for the detection of glass ( $88 \%$ vs $84 \% ; P=0.5183)$. The combination of all 3 images was no better than the combination of transmission and phase-contrast images ( $94 \%$ vs $96 \%, P=0.622)$.

\section{Sensitivity for Any Foreign Body}

Sensitivity for the detection of any foreign body combined was highest when transmission, dark-field, and phase-contrast images were viewed simultaneously ( $91 \%$, Table 1$)$. The combination of all 3 modalities was significantly more sensitive for the detection of any foreign body compared with transmission images alone (91\% vs $62 \%$; $P<0.0001)$ and compared with the combination of transmission and phase-contrast images $(91 \%$ vs $71 \%, P<0.0001)$. The difference in sensitivity between the combination of all 3 modalities and the combination of transmission and dark-field images was not significant (91\% vs $89 \%, P=0.5035)$.

\section{Specificity}

Specificity was $99 \%$ to $100 \%$ across all readers and radiography modalities (Table 1).

\section{DISCUSSION}

The results of our study indicate that adding dark-field and phase-contrast images substantially improve the detection of foreign bodies on radiographs compared with conventional (transmissionbased) radiography alone. A substantially increased sensitivity for the detection of wooden foreign bodies was found with dark-field images. This is consistent with theoretical considerations. Dark-field images visualize the strength of small-angle $\mathrm{x}$-ray scattering within a sample. Wood generates a strong dark-field signal due to small-angle x-ray scattering by its fibrous microstructure. Because of its rather homogenous internal structure, glass produced a very weak dark-field signal. Consequently, adding dark-field images alone did not significantly improve readers' sensitivity for glass foreign bodies.

For glass foreign bodies, a moderate increase in sensitivity was found when adding phase-contrast images. The differential phasecontrast images visualize phase-shifts of $\mathrm{x}$-ray beams occurring inside the probe. Such shifts occur predominantly at interfaces of materials with different refraction indices, which explain the edge-enhancing properties of phase-contrast images. The accentuation of the edges of glass fragments in phase-contrast images explains the increase in sensitivity.

A reader study with 5 blinded radiology residents, who were presented with various image combinations in 4 rounds of reading, was conducted. We chose to include transmission images in all 4 rounds and to investigate the effect of adding dark-field images, phase-contrast images, or both. The rationale for this study design was that gratingbased x-ray imaging inevitably produces a transmission image, too. If this imaging technique was adopted clinically, we believe that radiologists and other clinicians would always prefer to view dark-field and phase-contrast images in conjunction with the transmission image (which corresponds to a conventional radiograph). Therefore, we did not analyze the diagnostic performance of dark-field images or phase-contrast images alone but rather considered them as potential add-ons to transmission images.

The main limitation of our study is that the image acquisition time of approximately 3 minutes currently prohibits clinical application. The scan time can be substantially decreased by the use of large FOV gratings. These gratings are currently under development ${ }^{20}$ and will be included in future system updates.

For the purpose of this study, foreign bodies were placed on top of the skin of pig paws (rather than inside). This might be seen as another limitation of our study, because there could be differences in signal behavior compared with intracorporeal placement of the foreign bodies (extracorporeal foreign bodies are surrounded by air, not tissue). However, we do not expect different results regarding the overall sensitivity of dark-field radiography as the underlying physical principle does not change. Future experiments where foreign bodies placed within different types of soft tissue (= intracorporal foreign bodies) should be performed to proof this new hypothesis. This study should be conducted using human specimens rather that animal specimens. The promising results of the present experiments may be used to apply for ethical approval for such a study.

In conclusion, our results indicate that grating-based x-ray darkfield and phase-contrast imaging is a promising new technique, which substantially improves the detection of foreign bodies on radiographs. Adding dark-field images substantially improves the detection of wooden foreign bodies compared with the analysis of conventional (transmission) radiographs alone. Detection of glass foreign bodies is moderately improved when phase-contrast images are added.

\section{REFERENCES}

1. DeBoard RH, Rondeau DF, Kang CS, et al. Principles of basic wound evaluation and management in the emergency department. Emerg Med Clin North Am. 2007;25:23-39.

2. Steele MT, Tran LV, Watson WA, et al. Retained glass foreign bodies in wounds: predictive value of wound characteristics, patient perception, and wound exploration. Am J Emerg Med. 1998;16:627-630.

3. Potini VC, Francisco R, Shamian B, et al. Sequelae of foreign bodies in the wrist and hand. Hand (N Y). 2013;8:77-81.

4. Davis J, Czerniski B, Au A, et al. Diagnostic accuracy of ultrasonography in retained soft tissue foreign bodies: a systematic review and meta-analysis. Acad Emerg Med. 2015;22:777-787.

5. Anderson MA, Newmeyer WL 3rd, Kilgore ES Jr. Diagnosis and treatment of retained foreign bodies in the hand. Am J Surg. 1982;144:63-67.

6. Levine MR, Gorman SM, Young CF, et al. Clinical characteristics and management of wound foreign bodies in the ED. Am J Emerg Med. 2008;26:918-922.

7. Kaiser CW, Slowick T, Spurling KP, et al. Retained foreign bodies. J Trauma. 1997;43: $107-111$. 
8. Horton LK, Jacobson JA, Powell A, et al. Sonography and radiography of soft-tissue foreign bodies. AJR Am J Roentgenol. 2001;176:1155-1159.

9. Pfeiffer F, Bech M, Bunk O, et al. Hard-X-ray dark-field imaging using a grating interferometer. Nat Mater. 2008;7:134-137.

10. Yaroshenko A, Meinel FG, Bech M, et al. Pulmonary emphysema diagnosis with a preclinical small-animal x-ray dark-field scatter-contrast scanner. Radiology. 2013;269:427-433.

11. Schleede S, Meinel FG, Bech M, et al. Emphysema diagnosis using X-ray darkfield imaging at a laser-driven compact synchrotron light source. Proc Natl Acad Sci U S A. 2012;109:17880-17885.

12. Hellbach K, Yaroshenko A, Meinel FG, et al. In vivo dark-field radiography for early diagnosis and staging of pulmonary emphysema. Invest Radiol. 2015;50: $430-435$.

13. Hellbach K, Yaroshenko A, Willer K, et al. Facilitated diagnosis of pneumothoraces in newborn mice using x-ray dark-field radiography. Invest Radiol. 2016;51: $597-601$
14. Habbel C, Hetterich H, Willner M, et al. Ex vivo assessment of coronary atherosclerotic plaque by grating-based phase-contrast computed tomography: correlation with optical coherence tomography. Invest Radiol. 2017;52:223-231.

15. Notohamiprodjo S, Webber N, Birnbacher L, et al. Qualitative and quantitative evaluation of structural myocardial alterations by grating-based phase-contrast computed tomography. Invest Radiol. 2018;53:26-34.

16. Bech M, Tapfer A, Velroyen A, et al. In-vivo dark-field and phase-contrast X-ray imaging. Sci Rep. 2013;3:3209.

17. Malecki A, Eggl E, Schaff F, et al. Correlation of x-ray dark-field radiography to mechanical sample properties. Microsc Microanal. 2014;20:1528-1533.

18. Pfeiffer FWT, Bunk O, David CH. Phase retrieval and differential phase-contrast imaging with low-brilliance x-ray sources. Nat Phys. 2006;2:258-261.

19. McHugh ML. Interrater reliability: the kappa statistic. Biochem Med (Zagreb) 2012;22:276-282

20. Schröter TJ, Koch FJ, Meyer P, et al. Large field-of-view tiled grating structures for X-ray phase-contrast imaging. Rev Sci Instrum. 2017;88:015104. 